EUROPEAN ORGANIZATION FOR NUCLEAR RESEARCH

European Laboratory for Particle Physics

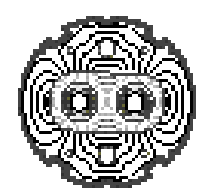

Large Hadron Collider Project

LHC Project Report 823

\title{
A Large Diameter Entrance Window for the LHC Beam Dump Line
}

\author{
B. Goddard, J-M. Jimenez, A. Presland, D. Ramos, R.Veness \\ CERN, Geneva, Switzerland
}

\begin{abstract}
The graphite LHC beam dump block TDE has to absorb the full LHC beam intensity at $7 \mathrm{TeV}$. The TDE vessel will be filled with inert gas at atmospheric pressure, and requires a large diameter entrance window for vacuum separation from the beam dumping transfer line. The swept LHC beam must traverse this window without damage for regular operation of the beam dump dilution system. For dilution failures, the entrance window must survive most of the accident cases, and must not fail catastrophically in the event of damage. The conceptual design of the entrance window is presented, together with the load conditions and performance criteria. The FLUKA energy deposition simulations and ANSYS stress calculations are described, and the results discussed.
\end{abstract}

Presented at PAC 2005, Knoxville, USA, May 16-20, 2005

CERN, CH-1211 Geneva 23, Switzerland

Geneva, June 2005 


\title{
A LARGE DIAMETER ENTRANCE WINDOW FOR THE LHC BEAM DUMP LINE
}

\author{
A. Presland, D. Ramos, B. Goddard, J-M. Jimenez, R. Veness, CERN, Geneva, Switzerland
}

\begin{abstract}
The graphite LHC beam dump block TDE has to absorb the full LHC beam intensity at $7 \mathrm{TeV}$. The TDE vessel will be filled with inert gas at atmospheric pressure, and requires a large diameter entrance window for vacuum separation from the beam dumping transfer line. The swept LHC beam must traverse this window without damage for regular operation of the beam dump dilution system. For dilution failures, the entrance window must survive most of the accident cases, and must not fail catastrophically in the event of damage. The conceptual design of the entrance window is presented, together with the load conditions and performance criteria. The FLUKA energy deposition simulations and ANSYS stress calculations are described, and the results discussed.
\end{abstract}

\section{INTRODUCTION}

The LHC beam dumping system [1] is designed to fastextract the beam in a loss-free way from each ring of the collider and transport it to an external dump block TDE, which is located in a cavern at the end of a separate $650 \mathrm{~m}$ long vacuum line. A system of horizontal and vertical dilution kickers $\mathrm{MKBH} / \mathrm{V}$ in this line will be used to sweep the beam in an "e" shaped form on the surface of the TDE.

A large $(600 \mathrm{~mm})$ diameter entrance window is required about $10 \mathrm{~m}$ upstream of the dump block, Fig. 1, at the end of each of the vacuum lines, to isolate the beam dump transfer lines, kept under high $\left(10^{-5} \mathrm{~Pa}\right)$ vacuum, from the beam dump TDE core which is kept under a slight $(0.2 \mathrm{kPa})$ overpressure of nitrogen. This TDE overpressure is to avoid any fire risk of the graphite dump block, which is routinely heated by 1000 degrees for a dump of the full beam, in the event of a containment failure.

The entrance window will therefore operate under a static differential pressure of more than one atmosphere, and needs a special mechanical design. This must maintain the required pressure differential, and also cope with both the repeated dynamic thermal load when the ultimate intensity LHC beam $\left(4.77 \times 10^{14}\right.$ protons at $\left.7 \mathrm{TeV}\right)$ is dumped, together with the worst-case thermal load which can happen if the dilution kicker system fails, either partially or totally. In these dilution failure cases the full LHC beam is swept over a much shorter length.

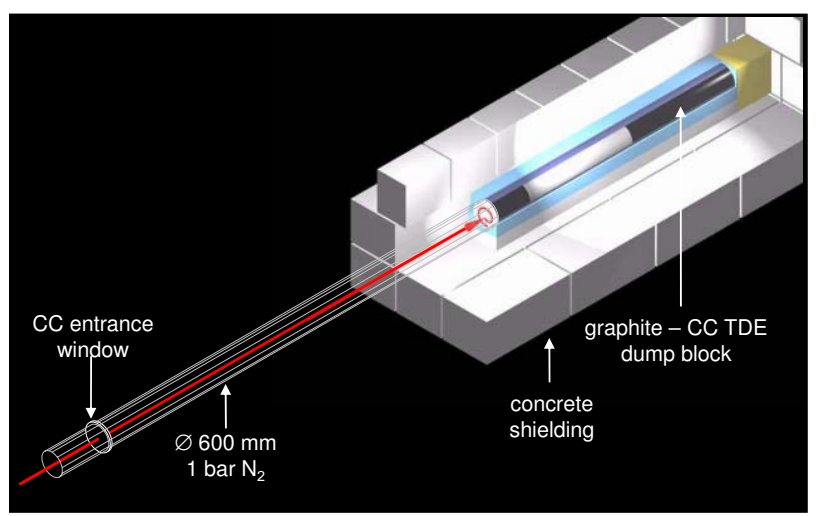

Figure 1: LHC dump block TDE and surrounding shielding, showing location of entrance window.

\section{LOAD CASES}

For the case of a normal beam dump, the 2808 proton bunches are swept over a path length of about $110 \mathrm{~cm}$ at the entrance window. The beta-functions at this location are about $4.6 \mathrm{~km}$ in each plane, such that the beam spot is circular, with a $\sigma$ of $6.0 \mathrm{~mm}$ at $450 \mathrm{GeV}$ and $1.5 \mathrm{~mm}$ at $7 \mathrm{TeV}$. The nominal sweep form is shown in Fig. 2 (for clarity the sweep is shown for $450 \mathrm{GeV}$ ).

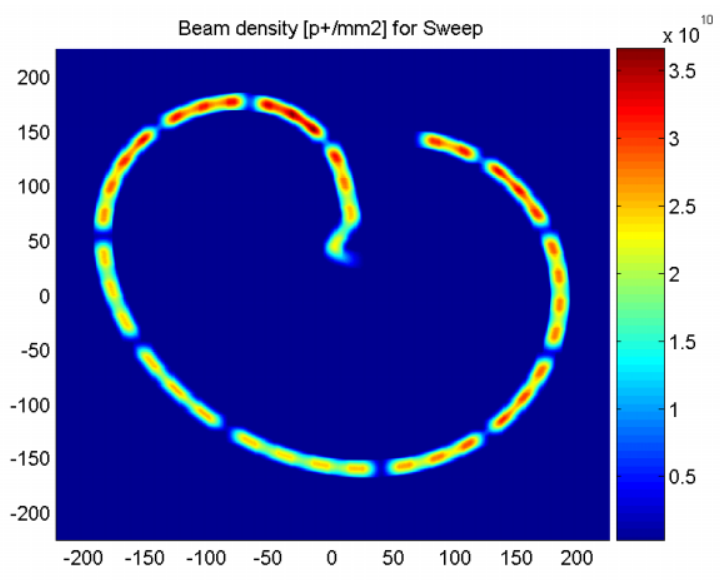

Figure 2: Nominal sweep profile $\left(\mathrm{p}+/ \mathrm{mm}^{2}\right)$ at TDE entrance window $(450 \mathrm{GeV}$ ultimate beam).

Failures of the dilution kickers MKB must not result in catastrophic damage to the entrance window. The most dangerous are the total failure of all the dilution kickers in one plane, e.g. Fig. 3, or a total dilution failure, in which 
the full beam impacts in a very localised area. Table 1 lists the maximum proton density at $7 \mathrm{TeV}$ for the various load cases, together with the rate expected per year from a reliability analysis of the system [2].

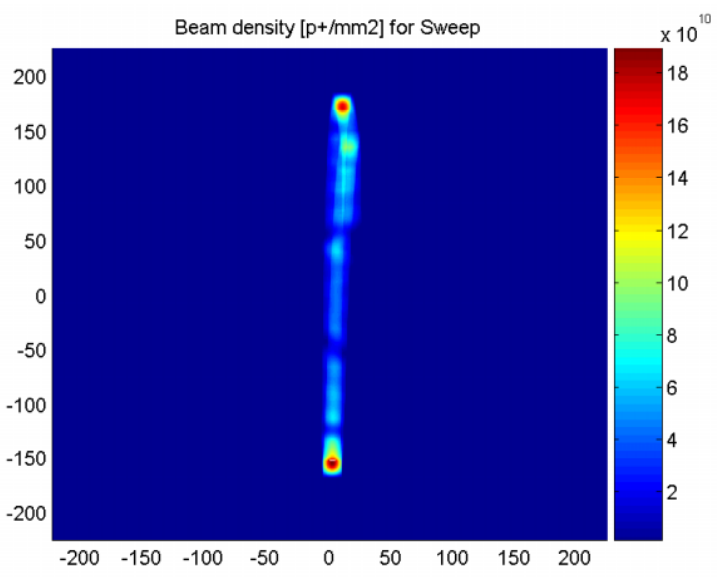

Figure 3: Sweep profile $\left(\mathrm{p}+/ \mathrm{mm}^{2}\right)$ at TDE entrance window, for all MKBH missing (450 GeV ultimate beam).

Table 1: Maximum $7 \mathrm{TeV}$ proton densities at the TDE entrance window for different load cases, together with expected occurrence rate.

\begin{tabular}{|l|c|c|c|}
\hline $\begin{array}{l}\text { Load case } \\
\text { (ultimate) }\end{array}$ & Total $\mathrm{p}+$ & $\begin{array}{c}\text { Max. density } \\
\left(\mathrm{p}+/ \mathrm{mm}^{2}\right)\end{array}$ & $\begin{array}{c}\text { Rate } \\
\left(\mathrm{y}^{-1}\right)\end{array}$ \\
\hline one bunch & $1.70 \times 10^{11}$ & $1.18 \times 10^{10}$ & - \\
nominal sweep & $4.77 \times 10^{14}$ & $1.64 \times 10^{11}$ & 400 \\
no MKBH & $4.77 \times 10^{14}$ & $1.41 \times 10^{12}$ & 0.008 \\
no MKBV & $4.77 \times 10^{14}$ & $1.78 \times 10^{12}$ & 0.005 \\
no MKB & $4.77 \times 10^{14}$ & $1.22 \times 10^{13}$ & $4.2 \times 10^{-5}$ \\
\hline
\end{tabular}

\section{MATERIALS CONSIDERATIONS AND CONCEPTUAL DESIGN}

The requirement that the entrance window withstand the dynamic load cases imposes the use of low- $Z$ materials for the mechanical functionality. The obvious choice is carbon-composite (CC), which has a density of around 1.7 $\mathrm{g} / \mathrm{cm}^{3}$, with excellent mechanical and thermal properties. However, the high permeability of $\mathrm{CC}$ means that an unacceptably high leak rate of $10 \mathrm{~Pa} \cdot \mathrm{m}^{3} . \mathrm{s}^{-1}$ (100 mbar.l.s $\left.{ }^{-1}\right)$ is expected for a $15 \mathrm{~mm}$ thickness. To meet the vacuum requirement an additional thin impermeable metallic layer is proposed. This will be on the high-pressure side of the window, such that the pressure differential keeps it pressed against the supporting CC plate - however, because of the high energy deposition expected in a metal, this layer will be the least likely to survive the dilution failures.

After some preliminary studies into suitable materials, the first-order design was based on a $15 \mathrm{~mm}$ thick CC plate, backed by a thin $200 \mu \mathrm{m}$ metallic foil. In a first instance a stainless-steel foil was chosen. The window would be contained in a short removable module with ring clamp flanges at either end, with the vacuum functionality assured by the foil, and the $\mathrm{CC}$ plate providing the mechanical stability, Figs. 4 and 5.

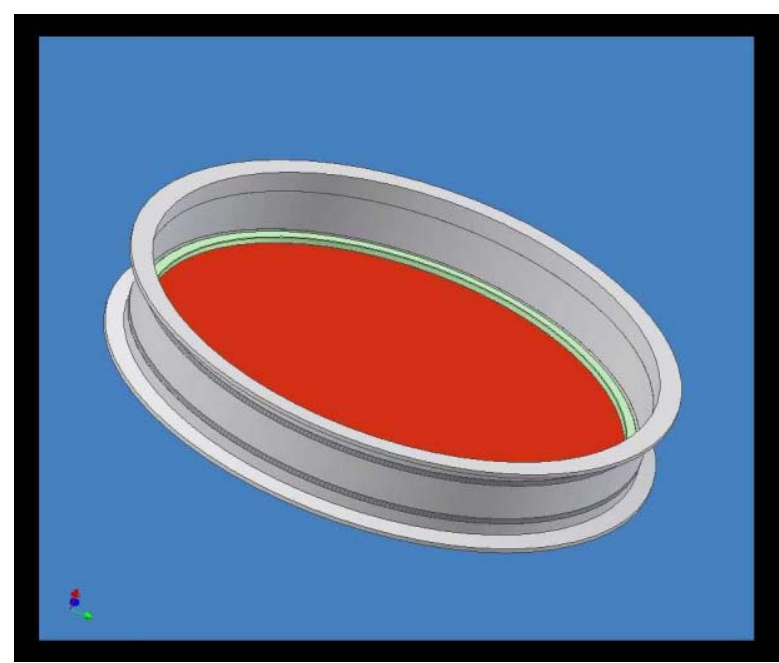

Figure 4: Schematic of entrance window module assembly.

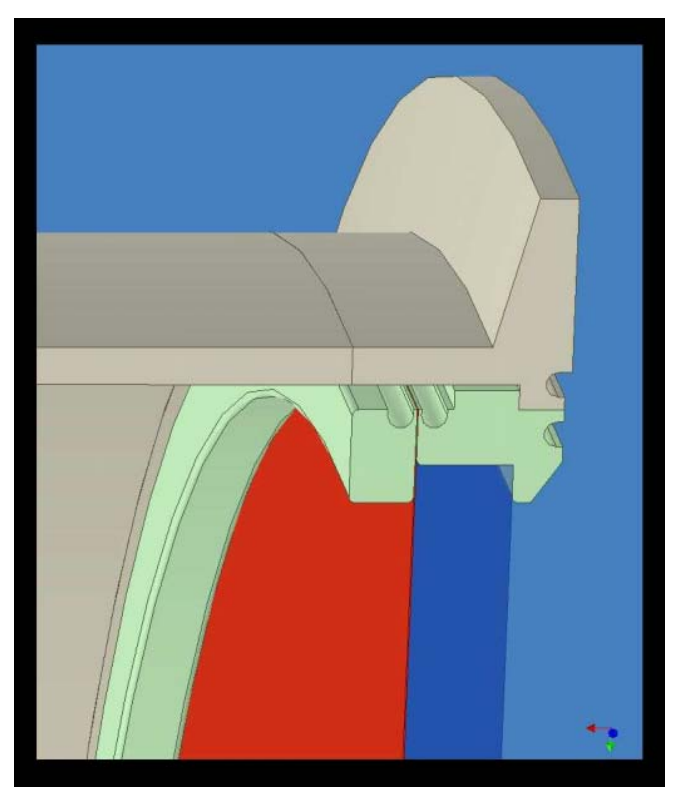

Figure 5: Detail of entrance window, showing CC plate, foil and vacuum flange.

\section{SIMULATION RESULTS}

The energy deposition in the $15 \mathrm{~mm}$ CC plate and $200 \mu \mathrm{m}$ steel foil was calculated with FLUKA [3] for a single LHC ultimate bunch. The overall temperature rise was calculated in a post-processing routine where this pattern was superimposed 2808 times, using the sweep profile. The temperature rise $\Delta \mathrm{T}$ was calculated numerically using the temperature dependant heat capacity $\mathrm{C}_{p}(\mathrm{~T})$. The results are shown in Fig. 6 for the nominal sweep. The maximum 
temperature rise is $15 \mathrm{~K}$ in the $\mathrm{CC}, 42 \mathrm{~K}$ in the foil. For the total dilution failure, the maximum temperature increases are $891 \mathrm{~K}$ and $3580 \mathrm{~K}$ respectively. The induced thermal stresses were calculated for the CC plate using the ANSYS FE code for the static combination of pressure and a simplified temperature profile, for the total dilution failure cases. The temperature rises and induced stresses are shown in Table 2 for the different load cases.

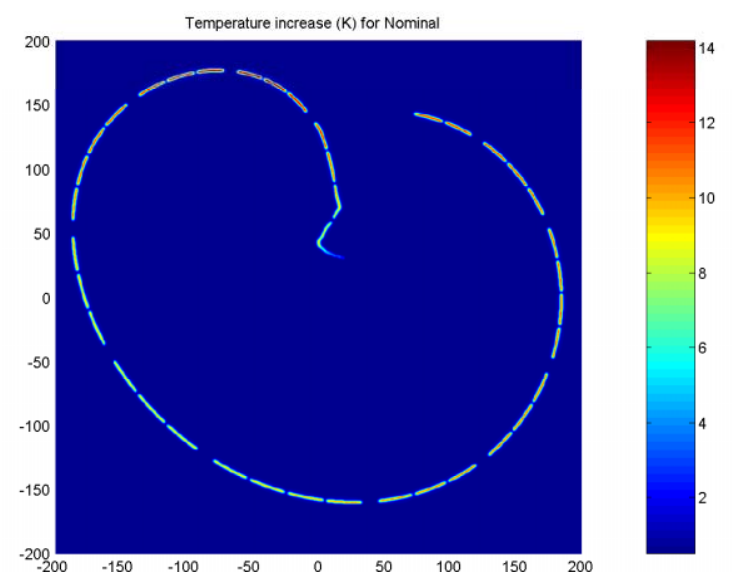

Figure 6: $\Delta \mathrm{T}$ for nominal sweep on $15 \mathrm{~mm}$ of $\mathrm{CC}(7 \mathrm{TeV}$ ultimate beam).

Table 2: $\Delta \mathrm{T}$ and maximum stresses in the $15 \mathrm{~mm} \mathrm{CC}$ and $200 \mu \mathrm{m}$ steel (SS), for different load cases.

\begin{tabular}{|l|r|r|r|}
\hline Load case & \multicolumn{2}{|c|}{$\Delta \mathrm{T}(\mathrm{K})$} & Stress $(\mathrm{MPa})$ \\
& $\mathrm{CC}$ & $\mathrm{SS}$ & $\mathrm{CC}$ \\
\hline & & & \\
one bunch & 1 & 3 & 0.2 \\
nominal sweep & 15 & 38 & - \\
no MKBH & 116 & 335 & - \\
no MKBV & 166 & 537 & - \\
no MKB & 891 & 3580 & 294 \\
\hline
\end{tabular}

The yield stress in the $\mathrm{CC}$ (at $1000{ }^{\circ} \mathrm{C}$ ) is around $320 \mathrm{MPa}$. The melting points of $\mathrm{CC}$ and SS are $\sim 4000{ }^{\circ} \mathrm{C}$ and $\sim 1400{ }^{\circ} \mathrm{C}$ respectively. Clearly, while it appears that the $\mathrm{CC}$ plate can survive all failure cases, the steel foil will be severely damaged for a total dilution failure. The stress effects of the partial sweep failures on the SS foil remain to be calculated; however, in view of the modest $\Delta \mathrm{T}$, these are not expected to pose a problem.

A total dilution failure will result in a major perforation of the steel foil [4], and hence a vacuum leak through the high permeability CC. The damaged area will be about $20 \times 5 \mathrm{~mm}$, Fig. 7. The entrance window will certainly need replacing; however, in the event of such a failure, the TDE dump block and downstream exit window are likely to be severely damaged, to an extent where a major intervention to replace the failed components will be necessary; the exchange of the entrance window under these circumstances will not an important factor in the recovery time.
Finally, the expected occurrence rate of such a failure is very low, Table 1 .

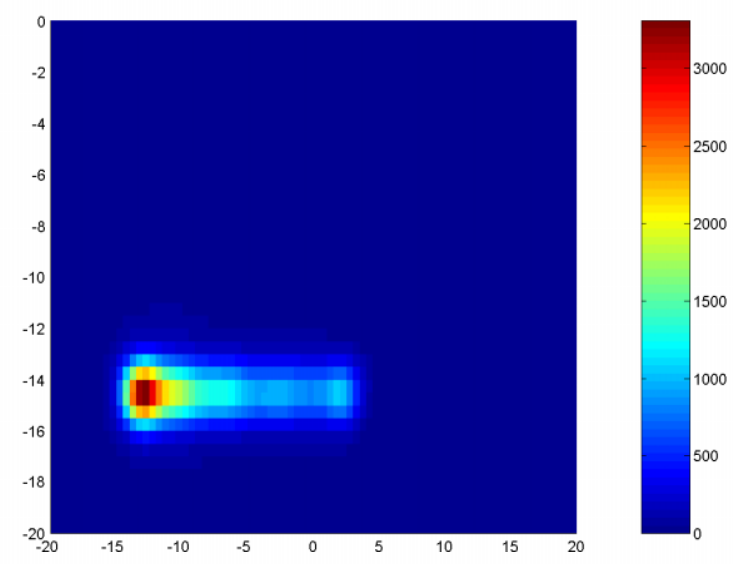

Figure 7: $\Delta \mathrm{T}$ for total dilution failure on a $200 \mu \mathrm{m}$ stainless steel foil, after $15 \mathrm{~mm}$ of CC (7 TeV ultimate beam).

\section{DISCUSSION}

The conceptual design study of the $600 \mathrm{~mm}$ diameter TDE entrance window has shown that a $15 \mathrm{~mm}$ CC plate will survive the worst-case dilution failure. The containment of the TDE block with respect to the TD vacuum line and the LHC machine vacuum should remain intact, although with a high leak rate through the CC.

The backing foil required to make the $\mathrm{CC}$ plate leak tight will, however, be severely damaged in the event of a total dilution failure, requiring the replacement of the window module. The design of the window connection, installation and alignment systems must take this eventuality into account, and the spares policy for this element must also be adequate. In case this situation is deemed unacceptable, an alternative solution could be developed which do not require the metal foil, such as two CC plates enclosing a short differential pumping region which would be kept at a low pressure (around $10^{-2} \mathrm{~Pa}$ ) with a suitable pump.

\section{ACKNOWLEDGEMENT}

The valuable input from $\mathrm{H}$. Kos and W. Weterings is gratefully acknowledged.

\section{REFERENCES}

[1] O.Brüning et al., LHC Design Report Volume III, http://ab-div.web.cern.ch/ab-div/Publications/LHCDesignReport.html

[2] R.Filippini, private communication.

[3] A.Fasso et al., FLUKA: Status and Prospective for Hadronic Applications, Proc. MonteCarlo 2000 Conference, Lisbon, pp.955-960, 2001.

[4] V.Kain et al., Material Damage Test with 450 GeV LHC-Type Beam, these proceedings. 